\title{
Chromosome 21 non-disjunction and Down syndrome birth in an Indian cohort: analysis of incidence and aetiology from family linkage data
}

\author{
SUJOY GHOSH ${ }^{1,2}$, PRANAMI BHAUMIK ${ }^{1}$, PRIYANKA GHOSH ${ }^{1}$ \\ AND SUBRATA KUMAR DEY ${ }^{1 *}$ \\ ${ }^{1}$ Human Genetics Research Unit, School of Biotechnology and Biological Sciences, West Bengal University of Technology, BF-142, Salt Lake \\ City, Sector I, Kolkata 700064, West Bengal, India \\ ${ }^{2}$ Department of Zoology, Sundarban Hazi Desarat College, South 24 Parganas, Pathankhali 743611, West Bengal, India
}

(Received 25 January 2010 and in revised form 29 March 2010)

\begin{abstract}
Summary
We analysed the family linkage data obtained from short tandem repeat (STR) genotyping of 212 unrelated Indian families having a single Down syndrome (DS) baby each, in order to explore the incidence and aetiology of this human aneuploidy in our cohort. The estimated values of maternal meiotic I and meiotic II non-disjunction (NDJ) errors of chromosome 21 (Ch 21) were $\sim 78$ and $\sim 22 \%$, respectively. Within the paternal outcome group, about 47 and $53 \%$ were accounted for NDJ at meiosis I and meiosis II, respectively. We estimated only $\sim 2 \%$ post-zygotic mitotic errors. The comparison of average age of conception between controls and DS-bearing mothers revealed a significant difference $(P<0 \cdot 001)$ with DS-bearing women were on an average older than controls and meiotic II non-disjoined mothers were oldest among meiotic outcome groups. Our linkage analysis suggested an overall reduction in recombination by more than $50 \%$ on meiotic I non-disjoined maternal Ch 21 with error prone to susceptible chiasma formation within the $\sim 5 \cdot 1 \mathrm{kbp}$ segment near the telomeric end. We stratified meiotic I non-disjoined women in three age groups, viz. young ( $\leqslant 28$ years), middle ( $29-34$ years) and old ( $\geqslant 35$ years) and found linear decrease in the frequency of achiasmate meiosis from the young to the old group. In contrary, a linear increase in the multiple chiasma frequency from the young to the old group was observed. Considering these results together, we propose that the risk factors for Ch 21 NDJ are of two types, one being 'maternal age-independent' and the other being 'maternal age-dependent'. Moreover, a comparison of our present Indian dataset with that of other published data of ethnically different populations suggested that the genetics that underlies the NDJ of Ch 21 is probably universal irrespective of racial difference across human populations. The present study is the first population-based report on any DS cohort from the Indian subcontinent and our work will help future workers in understanding better the aetiology of this birth defect.
\end{abstract}

\section{Introduction}

Down syndrome (DS), the most frequent live born aneuploidy in human, is predominantly caused by trisomy of chromosome 21 (Ch 21) and its aetiologic factors are under continuous scrutiny since its discovery by Lejeune et al. (1959). Several groups of

\footnotetext{
* Corresponding author. Human Genetics Research Unit, School of Biotechnology and Biological Sciences, West Bengal University of Technology, BF-142, Salt Lake City, Sector I, 700064, West Bengal, India. Tel: 033-2321-0731, Ext. 204. Fax: 033-2321-7578. e-mail: subrata.dey@wbut.ac.in
}

workers have tried to explore the factors associated with non-disjunction (NDJ) of $\mathrm{Ch} 21$ and have identified advanced maternal age (Hassold \& Chiu, 1985; Allen et al., 2009) and altered pattern of recombination of maternal non-disjoined chromosomes (Warren et al., 1987; Sherman et al., 1991; Oliver et al., 2008) as two strong correlates that affect proper segregation of chromosomes at oogenesis, particularly at first meiotic division (MI) (Sherman et al., 2007; Allen et al., 2009). In elucidating the proximate causes of this sex bias of the risk factors, two different hypotheses have been put forward. According to one 
school of thought (Sherman et al., 2007; Allen et al., 2009), the protracted phase of MI arrest in women that lasts for several years makes the oocyte more vulnerable to NDJ than spermatozoa. An alternative to this explanation came from the study of NDJ in Drosophila (Zwick et al., 1999b), the organism that does not experience MI arrest. This line of thinking emphasized the meiotic drive of chromosomes and subsequent natural selection in asymmetric meiosis in females as the probable reasons of sex biasness of NDJ.

The association of advanced maternal age with DS birth is still an enigma. Although advanced maternal age is not the cause of NDJ, it is an obvious risk of DS birth. Apart from the studies on the higher incidence of DS birth at an advanced maternal age, the impact of maternal aging on the generation of aneuploid pregnancy was assessed in spontaneously aborted fetus and oocytes (Pellestor et al., 2003; Yusuf \& Naeem, 2004). These studies suggested a steady rise in the proportion of trisomy conceptuses with increasing maternal age. To explain the age effect on the developing oocyte in relation to its decreasing efficiency to segregate homologous chromosomes properly, several hypotheses have been put forward. These include changes associated with the oocyte pool size, disturbance in ovarian hormone balance (EichenlaubRitter \& Boll, 1989; Gaulden, 1992; Warburton, 2005), sub-optimal operation of the spindle apparatus and age-related degradation of sister chromatid cohesion protein complexes (Schon et al., 2000; Eichenlaub-Ritter et al., 2004; Hodges et al., 2005; Steuerwald, 2005).

Recombination involves the formation of the chiasma that holds two non-sister chromatids together and ensures proper separation of homologous chromosomes to the opposite poles at anaphase I. For normally disjoined $\mathrm{Ch} 21$ during maternal meiosis, chiasma formation most often occurs in the middle of 21q (Lamb et al., 2005a). Any change in the normal recombination pattern due to altered chiasma placement is a risk of NDJ (Hassold et al., 1995; Koehler et al., 1996). The association of Ch 21 NDJ with an overall reduction of crossing-over and susceptible chiasma configuration was reiterated by different workers and subsequently confirmed in populationbased studies (Lamb et al., 2005a,b; Oliver et al., 2008; Ghosh et al., 2009). These studies included Ch 21-specific DNA markers analyses that facilitated the determination of parental origin of extra chromosome and helped to interpret the meiotic stage of NDJ unambiguously. Nevertheless, this approach enabled workers to monitor the pattern of recombination of the non-disjoined $\mathrm{Ch} 21$. Such population-based studies have suggested that maternal NDJ accounts for approximately $90 \%$ of the errors among live born free trisomy 21 cases (Mikkelsen et al., 1995; Yoon et al., 1996; Gómez et al., 2000; Sherman et al., 2005). Very recently, an interaction of maternal age with specific chiasma position has been reported (Oliver et al., 2008), which has identified single telomeric chiasma as a risk for maternal MI NDJ among younger women in contrast to single centromeric chiasma that increases the risk of Ch 21 NDJ at MII among older females.

We performed an initial study on DS sample population from the eastern part of India (Ghosh et al., 2009) which is ethnically, culturally and socioeconomically absolutely different from western populations on which the majority of aforementioned investigations were carried out. In that previous study, we analysed the position of individual chiasma on maternal non-disjoined chromosomes. Although our sample size was small, the results showed a significant shift in the chiasma position from telomere to centromere as a function of increasing maternal age. The smaller sample size did restrict our attempt to estimate the overall pattern of recombination throughout the length of the maternal non-disjoined chromosomes and to inspect the epidemiology of DS birth with respect to parental origin.

In the present study, we analysed the recombination pattern of maternal MI non-disjoined Ch 21 from family linkage data of relatively larger sample size and estimated the frequency of observed recombination events on maternal MI non-disjoined chromosomes. Further, we resolved the pattern of interaction between the numbers of observed chiasma on maternal MI NDJ chromosomes and maternal age of conception of trisomy 21 fetus. Moreover, our present study is focused to provide an epidemiological overview regarding the parental origin and parental age of conception of DS children in Indian cohort.

\section{Material and methods}

\section{(i) Subjects}

A total of 212 families, each with a single DS child having free trisomy 21 , were included in our study. Families were referred randomly from different Medical Colleges and Hospitals of Kolkata and adjoining areas. The families were unrelated and heterogeneous with respect to their religion, but were chiefly Bengali-speaking. A detailed family history with informed consent was taken from each participating family. The design of experiments with human tissue samples and subsequent data analyses were reviewed and approved by the institutional ethics committee constituted by the West Bengal University of Technology. Peripheral blood samples were collected from the father, mother and DS child. We included 130 healthy volunteer couples as controls, each having a healthy euploid child. 


\section{(ii) Cytogenetic analysis}

To include only the free trisomy 21 cases and exclude mothers with hidden mosaicism, conventional karyotyping was performed. At least 30 metaphase plates were analysed for each to confirm the chromosomal profiles of cases and controls.

\section{(iii) Genotyping and parental origin determination}

Genotyping was performed using 10 highly polymorphic STR markers spanning from the pericentromeric region to telomere of 21q. The order of markers was centromere - D21S1432 - D21S11 - D21S1437 - D21S1270 - D21S167 - D21S1412 - D21S2055 D21S1260 - D21S1411 - D21S1446 - qter. The maternal origin of NDJ was determined by establishing the contribution of maternal alleles to the DS child. Likewise, the paternal origin was also determined. The decision was considered confirmed when at least two markers were informative and the allelic status of the rest of the markers was consistent with that inference. We used additional four pericentric STR markers to determine the stage of meiotic origin of NDJ, i.e. MI errors or MII errors. The markers were: D21S369, D21S215, D21S258 and D21S120. We inferred an MI error when the parental heterozygosity of these markers was retained in the trisomic child ('non-reduced', N). If the parental heterozygosity was 'reduced' (R) to homozygosity in the trisomic child, we concluded that the case was of MII origin. We inferred post-zygotic mitotic error when all the markers remained 'reduced' without showing any detectable crossover event on non-disjoined chromosomes.

\section{(iv) Linkage analysis}

The linkage analysis was performed only for 131 informative maternal MI cases, as the rest of the meiotic outcome groups remained sub-optimal to do so. In other words, only the maternal MI category among all meiotic outcome groups met the minimum sample size requirement of the software (mentioned later) that was used to get an unbiased estimate of linkage. We scored the marker status as reduced (R), nonreduced $(\mathrm{N})$ or uninformative $(\mathrm{U})$ and arranged them successively in a direction from proximal to distal of centromere on 21q arm to make a 'data string' for each family. The recombination events were detected on a chromosome after observing the change of status of two successive markers, either $\mathrm{R} \rightarrow \mathrm{N}$ or $\mathrm{N} \rightarrow \mathrm{R}$ in a single family. The data sheet comprising all such family records was then analysed with 'NDJMap' software (Feingold et al., 2000), specially designed for analysing trisomy family data and we obtained the ' $y$ ' value which is the probability of recombination for each marker interval. This value was then subsequently converted to the recombination fraction ' $\theta$ ' and then to map the distance using the Kosambi map function. We scored recombination frequency in interval wise fashion, i.e., for example, the interval between the last centromeric marker D21S120 and the next marker D21S1432 was designated as interval 1, between D21S1432 and D21S11 as interval 2 and so on.

The control female map for the aforesaid STR markers was constructed directly from the $\mathrm{CEPH}$ genotyping database using the map-creating option at web site http://www.ensemble.org/Marshfield. We did this owing to a lack of STR genotyping database for Indian control population.

\section{(v) Statistical analysis}

In analysing the observed recombination in the trisomy-based map, two questions had been evaluated. Firstly, whether the overall recombination frequency had been reduced throughout the length of $21 \mathrm{q}$ arm in the trisomy map and secondly, whether the spatial distribution of crossing over would differ between the maps, i.e. whether the reduction of recombination equally affected the whole length of the long $\mathrm{arm}$. The association between reduced recombination and NDJ was evaluated comparing the overall genetic length of these two maps, assuming a constant ratio value ' $k$ ' between trisomy $\left(\mathrm{W}^{\mathrm{MI}}\right)$ to normal $\left(\mathrm{W}^{\mathrm{N}}\right)$ map length $\left(k=\mathrm{W}^{\mathrm{MI}} / \mathrm{W}^{\mathrm{N}}\right)$. If there was no association between recombination and NDJ, then the $k$ value should be 1 owing to the assumption that the genetic length of the trisomy-based map and female map would be similar at that condition. The trisomy map was then compared with the CEPH control map using the chi-square method. The average parental age of meiotic outcome groups was compared with the $t$-test. The epidemiological data were compared with other population data by Fisher's exact test. The comparison of the chiasma frequency among the age groups was done by a $2 \times 2$ test.

\section{Results}

\section{(i) Parental origin and MI:MII ratio}

The parental origin of non-disjoined Ch 21 was detected successfully in 201 families out of the participating 212 with a given STR marker set. The maternal meiotic error was recorded as $88.06 \%$ of all cases in contrast to $9.95 \%$ paternal and only $1.99 \%(\sim 2 \%)$ post-zygotic mitotic errors. We estimated $77.51 \%$ MI and $22.49 \%$ MII errors among all informative maternal origin cases. The ratio between these two maternal meiotic outcome groups was $3 \cdot 4: 1$. On the contrary, the estimated proportions of MI and MII 
Table 1. Origin of Trisomy 21 and their frequency in Indian cohort

\begin{tabular}{llrll}
\hline \hline \multirow{2}{*}{ Origin } & $\begin{array}{l}\text { Meiotic outcome } \\
\text { groups }\end{array}$ & $\begin{array}{l}\text { Sample } \\
\text { size }\end{array}$ & Proportions & $\begin{array}{l}\text { Frequency } \\
(\%)\end{array}$ \\
\hline Maternal & Meiosis I (MI) & 131 & MI/(MI + MII) $=131 / 169$ & $77 \cdot 51$ \\
& Meiosis II (MII) & 38 & MII/(MI + MII) $=38 / 169$ & $22 \cdot 49$ \\
& Stage unknown & 8 & & \\
& Subtotal & 177 & Maternal/All $=177 / 201$ & $88 \cdot 06$ \\
Paternal & Meiosis I (PI) & 8 & PI/(PI + PII) $=8 / 17$ & $47 \cdot 06$ \\
& Meiosis II (PII) & 9 & PII/(PI + PII) $=9 / 17$ & $52 \cdot 94$ \\
Stage unknown & 3 & & $9 \cdot 95$ \\
Post zygotic mitotic & Subtotal & 20 & Paternal/All $=20 / 201$ & $1 \cdot 99$ \\
Origin unknown & & 4 & $4 / 201$ & $5 \cdot 18$ \\
Total informative cases & & 11 & $11 / 212$ & \\
Total cases & & 201 & & \\
\hline \hline
\end{tabular}

Table 2. Distribution of average parental age of conception of DS child in Indian cohort

\begin{tabular}{lllll}
\hline \hline & & \multicolumn{2}{c}{$\begin{array}{l}\text { Parental age of conception } \\
\text { in year (mean } \pm \text { SD) }\end{array}$} \\
\cline { 3 - 4 } $\begin{array}{l}\text { Parent of } \\
\text { origin }\end{array}$ & $\begin{array}{l}\text { Meiotic outcome } \\
\text { groups }\end{array}$ & $\begin{array}{c}\text { Sample } \\
\text { size }\end{array}$ & Mother & Father \\
\hline Maternal & Meiosis I (MI) & 131 & $27 \cdot 62 \pm 5 \cdot 61$ & $33 \cdot 82 \pm 6 \cdot 77$ \\
& Meiosis II (MII) & 38 & $29 \cdot 02 \pm 2 \cdot 32$ & $34 \cdot 04 \pm 5 \cdot 21$ \\
Maternal & Meiosis I (PI) & 169 & $28 \cdot 11 \pm 6 \cdot 74$ & $34 \cdot 84 \pm 6 \cdot 89$ \\
& Meiosis II (PII) & 8 & $24 \cdot 57 \pm 3 \cdot 11$ & $30 \cdot 26 \pm 4 \cdot 12$ \\
Controls & PI+PII & 17 & $27 \cdot 01 \pm 5 \cdot 03$ & $34 \cdot 33 \pm 4 \cdot 14$ \\
\hline \hline
\end{tabular}

errors within paternal origin groups were $47 \cdot 06$ and $52.94 \%$, respectively, with a ratio value of almost $1: 1$ (Table 1).

\section{(ii) Parental age}

We estimated the average (mean \pm SD years) age of the conception of parents for all parental origin and meiotic outcome groups. We found a statistically significant difference $(P<0.001)$ in the mean age of conception between maternal MI group $(27 \cdot 62 \pm 5 \cdot 61$ years) and controls (24.02 \pm 4.41 years), as well as between maternal MII and controls $(29 \cdot 02 \pm 2 \cdot 32$ years; $P<0 \cdot 001)$. The estimated mean age (Table 2 ) for the combined MI and MII category did differ statistically from control too $(P<0 \cdot 001)$. The MII mothers did not exhibit any significant difference in the mean age of conception from the MI group, though the former was older by about 2 years from later. In analysing the paternal mean age of conception for paternal non-disjoined cases (Table 2), we found significant difference only between paternal MII and controls $(P=0.03)$; pair-wise comparisons for the rests remained insignificant.

\section{(iii) Linage analysis}

The estimated maternal MI trisomy map length was $30 \cdot 8$ centi-Morgan (cM), less than half the length of estimated CEPH control female genetic map for the same markers, which was $64.63 \mathrm{cM}$. The estimated ratio of $\mathrm{W}^{\mathrm{MI}}: \mathrm{W}^{\mathrm{N}}$ was $0 \cdot 47$, indicating an overall reduction in the map length of $\mathrm{Ch} 21 \mathrm{q}$. In addressing the second query as mentioned in the 'Statistical analyses' section, comparison was made between maternal MI-based trisomy map and normal $\mathrm{CEPH}$ map, to test whether the interval distance ratio was constant over the length of the chromosome arm. If the specific region of the trisomy map exhibited significantly high or low frequency of recombination than the control map, then the interval distance ratio values $(k)$ should vary among intervals. Using this approach, we observed (Table 3 ) that the distribution of recombination on $21 \mathrm{q}$ was significantly different between trisomy and control female map ( $P$ value was almost zero for chi-squared test), with more chiasma formation towards the telomere in non-disjoined map as evidenced by increased map length in peri-telomeric intervals (intervals 9 and 10). In contrast, the MI non-disjoined chromosomes 
Table 3. Comparison of genetic length (cM) of maker intervals of MI non-disjoined and control CEPH female maps

\begin{tabular}{|c|c|c|c|c|c|c|c|c|c|c|}
\hline \multirow[b]{2}{*}{ Types of map } & \multicolumn{10}{|c|}{ Marker intervals (centromere $\rightarrow$ telomere) on $21 \mathrm{q}$ and respective genetic length $(\mathrm{cM})$} \\
\hline & 1 & 2 & 3 & 4 & 5 & 6 & 7 & 8 & 9 & 10 \\
\hline MI Trisomy map & $1 \cdot 03$ & $4 \cdot 59$ & $3 \cdot 01$ & $2 \cdot 15$ & $3 \cdot 01$ & $1 \cdot 0$ & $2 \cdot 01$ & $6 \cdot 08$ & $5 \cdot 9$ & $2 \cdot 02$ \\
\hline Control CEPH map & $5 \cdot 26$ & $7 \cdot 04$ & $11 \cdot 76$ & $19 \cdot 99$ & $10 \cdot 79$ & $0 \cdot 97$ & $0 \cdot 39$ & $6 \cdot 24$ & $2 \cdot 15$ & $0 \cdot 04$ \\
\hline
\end{tabular}

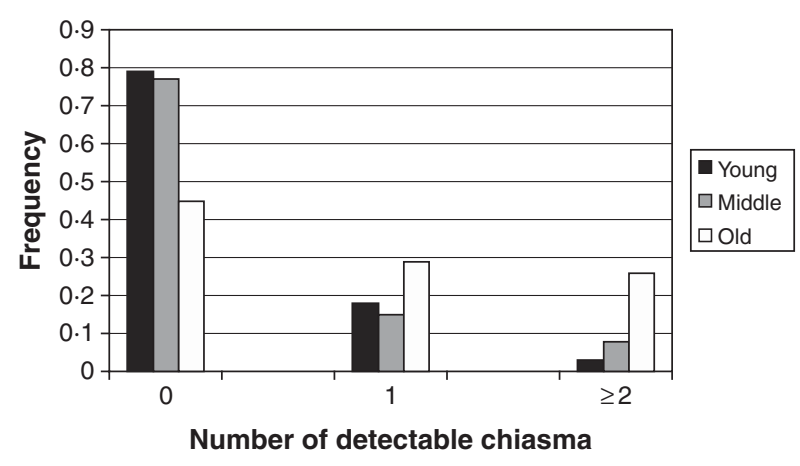

Fig. 1. Frequency of detectable chiasma on MI non-disjoined $\mathrm{Ch} 21$ among women of different age groups having DS babies.

exhibited a reduction in map length from centromereproximal to the middle part of ' $\mathrm{q}$ ' arm that suggests a decrease in chiasma frequency and recombination in that region.

\section{(iv) Maternal age effect on recombination frequency}

In evaluating the maternal age effect on the meiotic recombination and its risk to the aneuploid oocyte formation and subsequent trisomy 21 birth, we scored (Fig. 1) the frequency of detectable chiasma number on maternal MI NDJ chromosomes among different maternal age groups viz. young ( $\leqslant 28$ years), middle age (29-34 years) and old ( $\geqslant 35$ years). We observed that $\sim 79 \%$ (48 of 61 ) of young, $\sim 77 \%$ (30 of 39 ) of middle age and only $45 \%$ (14 of 31 ) of older women experienced achiasmate meioses. The frequencies of achiasmate meioses among age groups are significantly different for young versus old $(P=0 \cdot 00)$ and middle versus old $(P=0 \cdot 00)$. This tendency of decreasing proportion of non-recombinant aneuploid oocytes with age was almost linear and provides support to the model, which suggests that the risk of NDJ due to the absence of crossing over is operative during oogenesis irrespective of maternal age and for this we would expect to observe a greater proportion of achiasmate MI events among the younger mothers. This is because, for younger mothers, the absence of recombination is the only risk factor (under this simple model) in total risk scenario. Alternatively, the frequency of two or more observed crossover events increased linearly with age (Fig. 1); about 3\% of younger mothers ( 2 of 61 ) exhibited NDJ of multichiasmate chromosomes, whereas $8 \%$ of middle age ( 3 of 39 ) and $26 \%$ ( 8 of 31) of older women exhibited the same. Pair-wise comparison revealed that the distribution of multiple chiasmata as a function of maternal age was significantly different for young versus old $(P=0 \cdot 00)$ and middle versus old $(P=0 \cdot 001)$. This observation suggests that women experience risk for NDJ of Ch 21 even in the presence of multiple chiasmata at MI with increasing age.

\section{Discussion}

The aetiology of DS is still an enigma and its complete picture is yet to be deciphered. In the present study, we analysed the parental and meiotic origin of NDJ in a sample population of DS from Indian cohort and found that in majority of informative cases NDJ occurred during maternal gametogenesis $(88.06 \%)$, particularly at MI $(77 \cdot 51 \%)$. For the estimated $9.95 \%$ paternal cases, the MI and MII ratio value is almost 1:1 (Table 1). We were curious whether this epidemiological pattern of DS birth in Indian cohort is similar to that of other ethnically different populations reported earlier. We compared our Indian dataset with those of US (Allen et al., 2009), Spanish (Gómez et al., 2000), Ukraine and Russian cohorts (Machatkova et al., 2005), using Fisher's exact test. We did not obtain any significant difference in any pair-wise test for maternal:paternal ratio. But the results of our Indian maternal meiotic outcome groups differed statistically from the Spanish $(P=0.001)$ and Ukraine cohorts $(P=0 \cdot 02)$. No such difference was obtained for paternally originated cases. The difference between the Indian and other cohorts for maternal outcome groups might be due to the true ethnic difference among populations or it might arise from sampling variation. Considering the population size, the second possibility seemed to be more likely as the samples of Spanish $(N=38)$ and Ukraine $(N=32)$ populations were much smaller than the sample size of our present Indian population cohort $(N=201)$.

The results of parental age analyses exhibited significant difference for the average age of conception among maternal MI, MII and control in a pair-wise 
manner $(P<0.001$ for all). The MI and MII mothers are on average older from the control mothers by $\sim 3$ years and $\sim 5$ years, respectively (Table 2 ). The absence of statistical difference in pair-wise comparison for paternally originated cases suggests that the 'aging factor' may not be associated with NDJ at spermatogenesis. The only significant difference we obtained between paternal MII and control $(P=0 \cdot 03)$ may not be conclusive as the number of paternal cases was really small. This set of data suggests that advanced maternal age is a risk factor for NDJ of Ch 21 in our Indian population too. One notable aspect in our present Indian dataset is that the mothers are comparatively younger for their age of conception of DS fetus than those of the US cohort (Allen et al., 2009). This could be explained by either of the two possibilities. The first one includes a chance of real ethnic difference between populations for which Indian mothers are at risk of having aneuploid pregnancy at an earlier age than US mothers. The second possibility suggests that the difference is due to variation in social practice with an earlier age of conception in the Indian population.

The result of linkage analysis exhibited significant reduction in the map length of maternal MI nondisjoined $\mathrm{Ch} 21$, which in turn suggests that nonexchange chromosomes are at a risk of non-separation at the MI of oogenesis. This overall reduction in recombination is a probable universal risk for aberrant chromosome segregation as reiterated by several workers in their studies in humans (Lamb et al., 2005a; Freeman et al., 2007) and model organisms (Rockmill \& Roeder, 1994; Koehler \& Hassold, 1998; Stewart \& Dawson, 2004; Davis \& Smith, 2005). Reciprocal recombination during crossing over leads to that formation of chiasma that links the two homologues of bivalent with proper configuration and enables them to orient correctly and form stable attachments to both poles at MI. This stable attachment is essential for a precise and proper segregation of chromosomes during the first meiotic division (Smith \& Nicolas, 1998) and is achieved because of tension resulting from spindle forces from the two poles counteracted by attachments of homologues to each other (Wesley et al., 1992). Any deviation in this usual recombination profile may lead to malsegregation of respective chromosomes leading to an increased level of NDJ and subsequent aneuploid gamete formation (Lamb et al., 2005a).

In addition to the estimation of the overall recombination rate, we compared the genetic length of marker intervals of the MI NDJ map with that of $\mathrm{CEPH}$ controls map to inspect the change in the chiasma placement at different segments of the long arm of non-disjoined $\mathrm{Ch} 21$. We found a major reduction in chiasma frequency at the proximal and middle part of the chromosome arm (intervals 1 to 5 ;
Table 3), which covers $\sim 22.5 \mathrm{Mb}$ region. In contrast, we observed an increase in recombination frequency at telomere and its vicinity (intervals 9 and 10) covering $\sim 5.1 \mathrm{Mb}$ segment. Thus, a susceptible peritelomeric chiasma configuration is evident, which probably places the chromosomes at the risk of NDJ even when they do participate in recombination at MI. Probably this distal chiasma links the homologues less efficiently and oriented each kinetochore to opposing spindle poles. Most likely, this susceptibility is related to the minimal amount of sister-chromatid cohesion complex remaining distal to the exchange event (Orr-Weaver, 1996). The risk of single telomeric exchange for NDJ had also been reported from other eukaryotic model organisms (Zetka \& Ross, 1995; Koehler et al., 1996; Ross et al., 1996).

The result of the analysis involving interactions between maternal age and amount of meiotic crossover reinforces the model proposed earlier (Oliver et al., 2008; Ghosh et al., 2009) and clearly demonstrates that the absence of crossing over imposes a risk of chromosomal NDJ irrespective of maternal age. In our previous study (Ghosh et al., 2009), we did not find an expected linear decrease of non-recombinant aneuploid oocytes with increasing maternal age according to the proposed model. At that time, we scored a puzzling higher proportion of achiasmate meioses in the middle-age group and that was probably due to sampling error. By this time, we have increased our sample size and achieved an almost clear linear trend, with $79 \%$ achiasmate meiosis among young, $77 \%$ among middle age and $45 \%$ among old women (Fig. 1). This difference in the distribution of achiasmate meioses is statistically significant $(P=0.00$ for both young versus old and middle versus old) and seems to be real. This data confirms our model (Ghosh et al., 2009) which proposes that the lack of recombination is an age-independent threat for NDJ and it perturbs proper meiotic chromosome segregation stochastically. That is why we obtained a greater proportion of non-recombinant chromosomes among younger women for whom this is the only risk factor in the absence of any other age-related challenges. In addition, we observed a linear increase in the proportion of multi-chiasmate MI errors from younger to older women (Fig. 1) and the pair-wise difference in frequencies among maternal age groups was significant $(P=0.00$ for young versus old and $P=0.001$ for middle versus old). This finding is important as it directly supports the hypothesis that the number of exchanges may be protective against the maternal age-related risk factor. This was first suggested by Robinson et al. (1998), who found that among maternal MI chromosome 15 NDJ errors, the age of the mother was significantly increased among cases with multiple recombinants compared with those having zero or only one observed recombinant. 
Similar results were also obtained from analyses involving maternal NDJ of the $\mathrm{X}$ chromosome (Thomas et al., 2001) and chromosome 18 (Fisher et al., 1995). In contrary, the previous populationbased study for Ch 21 NDJ (Oliver et al., 2008) did not report such a trend. That result exhibited 40, 23 and $33 \%$ tetrads that experienced multiple exchanges among young, middle and older women, respectively, within the MI error category. In this regard, our observation is probably the first that provides support to this hypothesis for Ch 21 NDJ. Moreover, the observation suggests that instead of having multiple chiasmata, the chromosomes are also at the risk of NDJ among older women. But we need to be cautious in our conclusion about multiple chiasmata, due to our limitations in having less number of cases exhibiting multiple recombinants and comparatively less marker coverage outside the centromere and telomere.

Our result of parental age analysis suggests that advanced maternal age is a risk of aneuploid gamete formation. Several hypotheses have been put forward to explain the age-related genetic and molecular changes in the ovarian microenvironment that instigates aneuploid gamete formation among aged women. These include degradation of proteins that are involved in various sophisticated cytogenetic processes at the post-resumption phase after long MI arrest such as formation and activity of spindle apparatus (Hawley et al., 1994), sister-chromatid cohesion complex (Wolstenholme \& Angell, 2000), anaphase-promoting complex and/or the chromosome surveillance system (Cheslock et al., 2005). The last one is of special interest as it ensures proper separation of non-recombinant chromosomes at anaphase I and have also been reported in model organisms (Koehler \& Hassold, 1998; Zwick et al., 1999a; Davis \& Smith, 2005). Besides these, a 'biological aging' hypothesis was proposed (Warburton, 2005), which states that gradual chronological aging is associated with a decline in the total oocyte pool and number of maturing antral follicles that are recruited in each menstrual cycle. This decline in the number of follicles together with an accompanying decrease in the total oocyte pool generates a change in the hormonal environment of the ovary, which causes meiotic errors. In addition, the 'oocyte mosaicism selection' model proposed by Hulten et al. (2010) suggests that gradual accumulation of aneuploid oocytes with age and their random selection for ovulation are the cause of maternal age-related increased incidence of DS births. Very recently we have resolved the issue of maternal aging and its relation to DS birth at the molecular level by estimating the telomere length of the maternal age groups and have proposed the 'genetic aging hypothesis' (Ghosh et al., 2010), which suggests that the older mothers $(\geqslant 35$ years) who have DS babies are genetically older than the control mothers of the same chronological age. This 'genetic aging' is probably the background cause of all age-related aforementioned degenerative changes and malfunctions in the ovary.

In summary, our results provide evidence for the existence of two sets of factors that make Ch 21 vulnerable to NDJ at MI in oogenesis. The first set is 'maternal age-independent', happens stochastically that includes altered pattern of chiasma formation and reduction of meiotic recombination and is evident clearly among younger women. In contrary, the second set is 'maternal age-dependent', intensifies with age as exhibited by older women whose nondisjoined chromosomes experienced otherwise multiple chiasmata. We could not perform similar analyses for maternal MII cases, due to sub-optimal sample size. The outcome of our family linkage data are surprisingly concordant to that of other ethnically different population cohorts and it suggests the aetiology and genetics that underlie the process of NDJ of Ch 21 and subsequent DS birth may be similar across the human populations irrespective of their ethnic and socio-economic differences. Our study provides the first report regarding the incidence of DS birth in any Indian population cohort and brings us to a more explicit understanding of the risk factors associated with DS births.

We are extremely thankful to Professor Eleanor Feingold, Graduate School of Public Health, Pittsburgh University, Pennsylvania, USA for providing NDJMap program for linkage analysis of trisomy data and her valuable suggestions in statistical analyses. We would like to thank the families participated in the study and the professionals who helped us in collection of blood samples. The project was funded by University Grants Commission (UGC), New Delhi, India; Sanctioned No. F-3-111/2001 (SR-II).

\section{(i) Conflict of interest statement}

The authors declare no conflict of interest.

\section{References}

Allen, E. G., Freeman, S. B., Druschel, C., Hobbs, C. A., O'Leary, L. A., Romitti, P. A., Royle, M. H., Torfs, C. P. \& Sherman, S. L. (2009). Maternal age and risk for trisomy 21 assessed by the origin chromosome nondisjunction: a report from the Atlanta and National Down Syndrome Projects. Human Genetics 125, 41-52.

Cheslock, P. S., Kemp, B. J., Boumil, R. M. \& Dawson, D. S. (2005). The role of MAD1, MAD2 and MAD3 in meiotic progression and the segregation of non-exchange chromosomes. Nature Genetics 37, 756-760.

Davis, L. \& Smith, G. R. (2005). Dynein promotes achiasmate segregation in Schizosaccharomyces pombe. Genetics 170, 581-590.

Eichenlaub-Ritter, U. \& Boll, I. (1989). Nocodazole sensitivity, age-related aneuploidy, and alterations in the cell cycle during maturation of mouse oocytes. Cytogenetic Cell Genetics 52, 170-176. 
Eichenlaub-Ritter, U., Vogt, E., Yin, H. \& Gosden, R. (2004). Spindles, mitochondria and redox potential in ageing oocytes. Reproduction Biomedical Online 8, 45-58.

Fisher, J. M., Harvey, J. F., Morton, N. E. \& Jacobs, P. A. (1995). Trisomy 18: studies of the parent and cell division of origin and the effect of aberrant recombination on nondisjunction. American Journal of Human Genetics 56, 669-675.

Feingold, E., Brown, A. S. \& Sherman, S. L. (2000). Multipoint estimation of genetic maps for human trisomies with one parent or other partial data. American Journal of Human Genetics 66, 958-968.

Freeman, S. B., Allen, E. G., Oxford-Wright, C. L., Tinker, S. W., Druschel, C., Hobbs, C. A., O'Leary, L. A., Romitti, P. A., Royle, M. H., Torfs, C. P. \& Sherman, S. L. (2007). The National Down Syndrome Project: design and implementation. Public Health Report 122, 62-72.

Gaulden, M. E. (1992). Maternal age effect: the enigma of Down syndrome and other trisomic conditions. Mutation Research 296, 69-88.

Ghosh, S., Feingold, E., Chakraborty, S. \& Dey, S. K. (2010). Telomere length is associated with types of chromosome 21 nondisjunction: a new insight into the maternal age effect on Down syndrome birth. Human Genetics 127, 403-409.

Ghosh, S., Feingold, E. \& Dey, S. K. (2009). Etiology of Down syndrome: evidence for consistent association among altered meiotic recombination, nondisjunction, and maternal age across populations. American Journal Medical Genetics A 149A, 1415-1420.

Gómez, D., Solsona, E., Guitart, M., Baena, N., Gabau, E., Egozcue, J. \& Caballín, M. R. (2000). Origin of trisomy 21 in Down syndrome cases from a Spanish population registry. Annals of Genetics 43, 23-28.

Hassold, T. \& Chiu, D. (1985). Maternal age-specific rates of numerical chromosome abnormalities with special reference to trisomy. Human Genetics 70, 11-17.

Hassold, T., Merrill, M., Adkins, K., Freeman, S. \& Sherman, S. (1995). Recombination and maternal agedependent nondisjunction: molecular studies of trisomy 16. American Journal of Human Genetics 57, 867-874.

Hawley, R. S., Frazier, J. A. \& Rasooly, R. (1994). Separation anxiety: the etiology of nondisjunction in flies and people. Human Molecular Genetics 3, 1521-1528.

Hodges, C. A., Revenkova, E., Jessberger, R., Hassold, T. J. \& Hunt, P. A. (2005). SMC1beta-deficient female mice provide evidence that cohesins are a missing link in age-related nondisjunction. Nature Genetics 37, 1351-1355.

Hulten, M., Patel, S., Jonasson, J. \& Iwarsson, E. (2010). On the origin of the maternal age effect in trisomy 21 Down syndrome: the Oocyte Mosaicism Selection (OMS) model. Reproduction 139, 1-9.

Koehler, K. E. \& Hassold, T. J. (1998). Human aneuploidy: lessons from achiasmate segregation in Drosophila melanogaster. Annals of Human Genetics 62, 467-479.

Koehler, K. E., Hawley, R. S., Sherman, S. \& Hassold, T. (1996). Recombination and nondisjunction in humans and flies. Human Molecular Genetics 5, 1495-1504.

Lamb, N. E., Sherman, S. L. \& Hassold, T. J. (2005a). Effect of meiotic recombination on production of aneuploid gametes in human. Cytogenetics and Genome Research 111, 250-255.

Lamb, N. E., Yu, K., Shaffer, J., Feingold, E. \& Sherman, S. L. $(2005 b)$. Association between maternal age and meiotic recombination for trisomy 21. American Journal of Human Genetics 76, 91-99.
Lejeune, J., Turpin, R. \& Gautier, M. (1959). Mongolism; a chromosomal disease (trisomy). Bulletin de l'Académie Nationale de Médecine 143, 256-265.

Machatkova, M., Brouckova, M., Matejckova, M., Krebsova, A., Sperling, K., Vorsanova, S., Kutsev, S., Zerova, T., Arbuzova, S., Krejci, R., Petersen, M. \& Macek, M. Sr. (2005). QF-PCR examination of parental and meiotic origin of trisomy 21 in Central and Eastern Europe. Journal of Histochemistry and Cytochemistry 53, 371-373.

Mikkelsen, M., Hallberg, A., Poulsen, H., Frantzen, M., Hansen, J. \& Petersen, M. B. (1995). Epidemiology study of Down's syndrome in Denmark, including family studies of chromosomes and DNA markers. Development and Brain Dysfunction 8, 4-12.

Oliver, T. R., Feingold, E., Yu, K., Cheung, V., Tinker, S., Yadav-Shah, M., Masse, N. \& Sherman, S. L. (2008). New insight into human nondisjunction of chromosome 21 in oocyte. PloS Genetics 4, e1000033.

Orr-Weaver, T. (1996). Meiotic nondisjunction does the two-step. Nature Genetics 14, 374-376.

Pellestor, F., Andreo, B., Arnal, F., Humeau, C. \& Demaille, J. (2003). Maternal aging and chromosomal abnormalities: new data drawn from in vitro unfertilized human oocytes. Human Genetics 112, 195-203.

Robinson, W. P., Kuchinka, B. D., Bernasconi, F., Petersen, M. B., Schulze, A., Brondum-Nielsen, K., Christian, S. L., Ledbetter, D. H., Schinzel, A. A., Horsthemke, B., Schuffenhauer, S., Michaelis, R. C., Langlois, S. \& Hassold, T. J. (1998). Maternal meiosis I non-disjunction of chromosome 15: dependence of the maternal age effect on level of recombination. Human Molecular Genetics 7, 1011-1019.

Rockmill, B. \& Roeder, G. S. (1994). The Yeast medl mutant undergoes both meiotic homologue nondisjunction and precocious separation of sister chromatids. Genetics 136, 65-74.

Ross, L. O., Maxfield, R. \& Dawson, D. (1996). Exchanges are not equally able to enhance meiotic chromosome segregation in yeast. Proceeding of National Academy of Sciences of the USA 93, 4979-4983.

Schon, E. A., Kim, S. H., Ferreira, J. C., Magalhães, P., Grace, M., Warburton, D. \& Gross, S. J. (2000). Chromosomal non-disjunction in human oocytes: is there a mitochondrial connection? Human Reproduction 2, $160-172$.

Sherman, S. L., Allen, E. G., Bean, L. \& Freeman, S. B. (2007). Epidemiology of Down syndrome. Mental Retardation and Developmental Disability 13, 221-227.

Sherman, S. L., Freeman, S. B., Allen, E. G. \& Lamb, N. E. (2005). Risk factors for nondisjunction of trisomy 21. Cytogenetics and Genome Research 111, 273-280.

Sherman, S. L., Takaesu, N., Freeman, S. B., Grantham, M., Phillips, C., Blackstone, R. D., Jacobs, P. A., Cockwell, A. E., Freeman, V., Uchida, I., Mikkelsen, M., Kurnit, D. M., Buraczynska, M., Keats, B. J. B. \& Hassold, T. J. (1991). Trisomy 21: association between reduced recombination and nondisjunction. American Journal of Human Genetics 49, 608-620.

Smith, K. N. \& Nicolas, A. (1998). Recombination at work for meiosis. Current Opinion in Genetics and Development 8, 200-211.

Steuerwald, N. (2005). Meiotic spindle checkpoints for assessment of aneuploid oocytes. Cytogenetics and Genome Research 111, 256-259.

Stewart, M. N. \& Dawson, D. S. (2004). Potential roles for centromere pairing in meiotic chromosome segregation. Cell Cycle 3, 1232-1234. 
Thomas, N. S., Ennis, S., Sharp, A. J., Durkie, M., Hassold, T. J., Collins, A. R. \& Jacobs, P. A. (2001). Maternal sex chromosome non-disjunction: evidence for $\mathrm{X}$ chromosome-specific risk factors. Human Molecular Genetics $\mathbf{1 0}$, 243-250.

Warburton, D. (2005). Biological aging and the etiology of aneuploidy. Cytogenetics and Genome Research 111, 266-272.

Warren, A. C., Chakravarti, A., Wong, C., Slaugenhaupt, S. A., Halloran, S. L., Watkins, P. C., Metaxotou, C. \& Antonarakis, S. E. (1987). Evidence for reduced recombination on the nondisjoined chromosomes 21 in Down syndrome. Science 237, 652-654.

Wesley, Y., Miyazaki Terry, L. \& Orr-Weaver. (1992). Sister-chromatid misbehavior in Drosophila ord mutants. Genetics 342, 1047-1061.

Wolstenholme, J. \& Angell, R. R. (2000). Maternal age and trisomy-a unifying mechanism of formation. Chromosoma 109, 435-438.

Yoon, P. W., Freeman, S. B., Sherman, S. L., Taft, L. F., Gu, Y., Petty, D., Flanders, W. D., Khoury, M. J. \&
Hassold, T. J. (1996). Advanced maternal age and the risk of Down syndrome characterized by meiotic stage of chromosomal error: a population-based study. American Journal of Human Genetics 58, 628-633.

Yusuf, R. Z. \& Naeem, R. (2004). Cytogenetic abnormalities in products of conception: a relationship revisited. American Journal of Reproduction and Immunology 52, 88-96.

Zetka, M. C. \& Ross, A. M. (1995). Mutant rec-1 eliminates the meiotic pattern of crossing over in Caenorhabditis elegans. Genetics 141, 1339-1349.

Zwick, M. E., Cutler, D. J. \& Langley, C. H. (1999a). Classic Weinstein: tetrad analysis, genetic variation and achiasmate segregation in Drosophila and humans. Genetics 152, 1615-1629.

Zwick, M. E., Salstrom, J. L. \& Langley, C. H. (1999b). Genetic variation in rates of nondisjunction: association of two naturally occurring polymorphisms in the chromokinesin nod with increased rates of nondisjunction in Drosophila melanogaster. Genetics 152, $1605-1614$. 